\title{
Induction of PR-1 Proteins and Potentiation of Pathogen Signals by Salicylic Acid Exhibit the Same Dose Response and Structural Specificity in Plant Cell Cultures
}

\author{
Zhixin Xie, Baofang Fan, and Zhixiang Chen \\ Department of Microbiology, Molecular Biology and Biochemistry, University of Idaho, Moscow, Idaho \\ 83844-3052, U.S.A. \\ Accepted 20 February 1998.
}

\begin{abstract}
Based on the marked difference in both dose response and structural specificity, it has been recently proposed that the induction of acquired resistance and pathogenesisrelated (PR) proteins in intact plant leaves and the potentiation of pathogen signals in plant cell cultures by salicylic acid (SA) and its analogues are mediated by different SA signaling pathways initiated from different SA receptor systems. We show here, however, that the induction of PR1 proteins, the most widely used marker of acquired resistance, by SA and its analogues in tobacco cell cultures exhibits the same dose response and structural specificity as the potentiation of pathogen signals demonstrated in soybean cell cultures. The different dose response and structural specificity for the induction of PR-1 proteins by SA between intact tobacco leaves and cell cultures appear to be caused largely by the difference in uptake and/or metabolism of these chemical inducers by different types of plant cells. These results suggest that the potentiation of pathogen signals by SA, which may contribute greatly to SA function, does not involve a different, more potent signaling pathway from the one(s) responsible for the induction of acquired resistance and PR protein accumulation.
\end{abstract}

Additional keywords: hypersensitive response, systemic acquired resistance, 2,6-dihydroxybenzoic acid.

An attempted infection by an avirulent pathogen often results in activation of localized hypersensitive response in infected cells, as well as resistant mechanisms in the surrounding, and even distal, uninfected parts of the plant to establish systemic acquired resistance (Baker et al. 1997; Ryals et al. 1996; Yang et al. 1997). During the last several years, extensive studies have established that salicylic acid (SA) plays an essential role in the establishment of systemic acquired resistance induced by a variety of pathogens or pathogen elicitors (Cao et al. 1997; Ryals et al. 1996, 1997). Genetic and molecular studies have indicated that SA also participates in the

Corresponding author: Zhixiang Chen; Telephone: 1-208-885-4030; Fax: 1-208-885-6518; E-mail: zchen@uidaho.edu development of the localized hypersensitive disease resistance response induced by pathogen infection (Levine et al. 1994; Shirasu et al. 1997; Weymann et al. 1995).

Because SA affects a variety of biochemical and molecular events associated with enhanced disease resistance, a question is often raised as to whether there is one or several SA signal transduction pathways mediating various defense or defenserelated mechanisms. For example, application of exogenous $\mathrm{SA}$ at concentrations of 1 to $5 \mathrm{mM}$ has been long known to induce pathogenesis-related (PR) gene expression and acquired resistance against a variety of microbial pathogens (Malamy et al. 1996; Ward et al. 1991). Like SA, certain benzoic acid derivatives including acetylSA and 2,6-dihydroxybenzoic acid are able to induce both PR gene expression and acquired resistance with a similar potency (Antoniw and White 1980; Chen et al. 1993; White 1979). More recently, low concentrations ( 10 to $100 \mu \mathrm{M}$ ) of SA have been shown to potentiate pathogen-induced defense gene expression, $\mathrm{H}_{2} \mathrm{O}_{2}$ accumulation and hypersensitive cell death in plant suspension cultures (Levine et al. 1994; Shirasu et al. 1997). When compared with the induction of acquired resistance and PR gene expression in intact plant tissues, the potentiation of these pathogen-induced defense responses in cell cultures requires lower SA concentrations and exhibits difference in structural specificity with SA analogues. Based on the difference in dose response, it has been proposed that the induction of PR proteins and acquired resistance observed in intact plant tissues with relatively high concentrations of SA represents a coarse inductive mechanism while the defense response potentiation observed in cell suspension cultures by relatively low concentrations of SA represents a fine control mechanism for the action of SA in plant disease resistance (Shirasu et al. 1997). Because of the difference in structural specificity with SA analogues, it has been further suggested that these two types of biological effects are mediated by different SA signal transduction pathways, probably involving different SA receptor systems (Shirasu et al. 1997).

Since assays for these different SA-induced defense responses are carried out in different plants with different types of plant cells, the observed difference in the dose response and 
structural specificity could be caused by the difference between different plants or the difference in uptake and/or metabolism of these chemical inducers by different types of plant cells, rather than the action of different SA signal transduction pathways. To examine the potential effects of uptake and/or metabolism by different types of plant cells on the assays of SA-induced plant defense responses, we have compared intact tobacco leaves with tobacco cell cultures for the induction of PR-1 proteins, the most widely used marker for biotically and abiotically induced acquired resistance (Ryals et al. 1996), by SA and its structural analogues. Since the induction of PR-1 protein accumulation by SA and its analogues in intact tobacco leaves exhibit a marked difference in both dose response and structural specificity from the potentiation of pathogen-induced hypersensitive disease resistance responses observed in plant cell cultures, we were interested in determining whether a similar difference could still be observed when the induction of PR-1 protein accumulation was assayed in tobacco cell cultures.

We first examined the levels of PR-1 proteins in tobacco leaves after spraying with various concentrations of SA. As shown in Figure 1A, although PR-1 proteins started to accumulate in tobacco leaves after spraying with $0.5 \mathrm{mM} \mathrm{SA}$, high levels of PR-1 protein accumulation were observed only in tobacco leaves that had been treated with 1 to $5 \mathrm{mM}$ SA. Thus, as previously reported (Ward et al. 1991), the induction of PR-1 protein accumulation in tobacco leaves requires relatively high concentrations of SA. However, when the same experiment was performed in tobacco cell cultures, it was found that much lower concentrations of SA were required for the induction of high levels of PR-1 protein accumulation (Fig. 1B). Indeed, the optimum concentrations of SA for the induction of PR-1 protein accumulation in tobacco cell cultures $(5$ to $100 \mu \mathrm{M})$ were very similar to those $(10$ to $100 \mu \mathrm{M})$ required for potentiating pathogen-induced defense gene expression, $\mathrm{H}_{2} \mathrm{O}_{2}$ accumulation, and hypersensitive cell death observed in soybean cell cultures (Shirasu et al. 1997).

On sprayed tobacco leaves, SA solutions do not cover the entire surface because of the high water tension and waxy leaf surface, and dry out within the first hour after spraying. Furthermore, SA may not readily penetrate the cuticle of plant leaves as found with other highly polar molecules (Baur et al. 1997). Therefore, the much higher optimum concentrations for PR-1 protein accumulation in tobacco leaves might be due to the low rates of uptake of SA. To test this possibility, we examined whether floating tobacco leaf disks on SA solutions, which would facilitate SA uptake, might reduce the effective SA concentrations required for the induction of high levels of PR-1 protein accumulation. Indeed, if this modified method was used for treating tobacco leaves, only 50 to $200 \mu \mathrm{M}$ of SA was required to induce high levels of PR-1 protein accumulation (Fig. 1C). In fact, SA at a concentration as low as $5 \mu \mathrm{M}$ was found to induce a relatively high level of PR protein accumulation (Fig. 1C). Thus, the requirement of high levels of SA for induction of PR-1 proteins in sprayed tobacco leaves may be largely attributable to the poor uptake of SA.

Previous studies have shown that certain SA analogues, including acetylSA and 2,6-dihydroxybenzoic acid, are able to induce acquired resistance and PR gene expression (Chen et al. 1993). Interestingly, for potentiation of pathogen-induced defense gene expression, $\mathrm{H}_{2} \mathrm{O}_{2}$ accumulation, and hypersensi- tive cell death in soybean cell cultures, only SA and acetylSA, but not 2,6-dihydroxybenozic acid, have been found to be biologically active (Shirasu et al. 1997). In the present study, we confirmed that both acetylSA and 2,6-dihydroxybenzoic acid were able to induce PR-1 protein accumulation in tobacco leaves (Fig. 2). In contrast, 3-hydroxybenzoic acid, 4hydroxybenzoic acid, 2,4-hydroxybenzoic acid, and 3,4-dihydroxybenzoic acid were ineffective in tobacco leaves (Fig. 2 ). In tobacco cell cultures, the structural specificity with these SA analogues for induction of PR-1 protein accumulation was generally similar to that found with tobacco leaves. SA and acetylSA were both effective in inducing PR-1 protein accumulation in tobacco cell cultures and the five other benzoic acid derivatives that failed to induce PR-1 protein accumulation in intact tobacco leaves were also ineffective in cell suspension cultures (Fig. 2). The only exception was 2,6dihydroxybenzoic acid, which has been previously found to be unable to potentiate pathogen signals in soybean cell culture

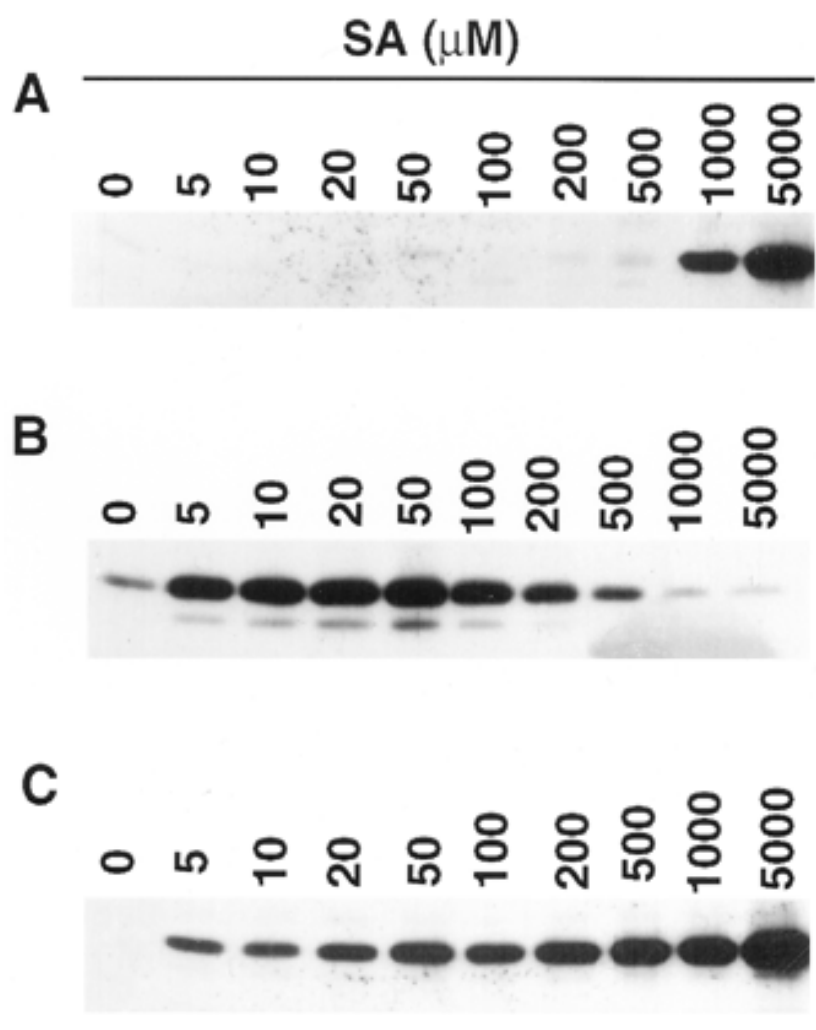

Fig. 1. Dose responses of induction of PR-1 protein accumulation by salicylic acid (SA) in intact tobacco leaves and cell cultures. A, Tobacco leaves sprayed with SA. B, Tobacco cell cultures incubated with SA. C, Tobacco leaf disks floated on SA solutions. Soluble proteins were extracted $20 \mathrm{~h}$ after treatment from tobacco leaves or cell cultures in buffer containing $50 \mathrm{mM}$ Tris/HCl, pH 7.5, $1 \mathrm{mM}$ EDTA, $10 \mathrm{mM} \beta$-mercaptoethanol and $10 \mu \mathrm{g}$ of phenylmethylsulfonyl fluoride per ml. Proteins were separated on a $15 \%$ sodium dodecyl sulfate-polyacrylamide gel and then transferred onto a nitrocellulose membrane. Monoclonal antibody 33G1, which specifically recognizes tobacco acidic PR-1 proteins (Chen et al. 1993), was used to detect PR-1 proteins. Tobacco cell cultures were grown at room temperature in the dark in MS medium supplemented with $1 \mu \mathrm{g}$ of $\alpha$-naphthaleneacetic acid per ml, $0.1 \mu \mathrm{g}$ of 2,4dichlorophenoxyacetic acid per $\mathrm{ml}$, and $0.1 \mu \mathrm{g}$ of benzyladenine per $\mathrm{ml}$. Cells were maintained by 10 -fold dilution with fresh medium every 5 to 6 days. Cells 2 days after dilution were used for determining induction of PR-1 protein accumulation. 
(Shirasu et al. 1997). 2,6-Dihydroxybenzoic acid failed to induce PR-1 accumulation in tobacco cell cultures although it is a strong inducer for accumulation of the same protein in tobacco leaves (Fig. 2).

The observed difference in the activity of 2,6-dihydroxybenzoic acid in inducing PR-1 protein accumulation in intact tobacco leaves versus cell suspension cultures could indicate the existence of different signal transduction pathways responsible for inducible PR-1 protein accumulation in different types of tobacco cells. Alternatively, it could be caused by the difference in uptake and/or metabolism of the inducer, which may be directly examined by monitoring its intracellular levels during the incubation with plant cells. This direct approach has been used for determining uptake and metabolism of SA by plant cells since SA can be readily separated from other
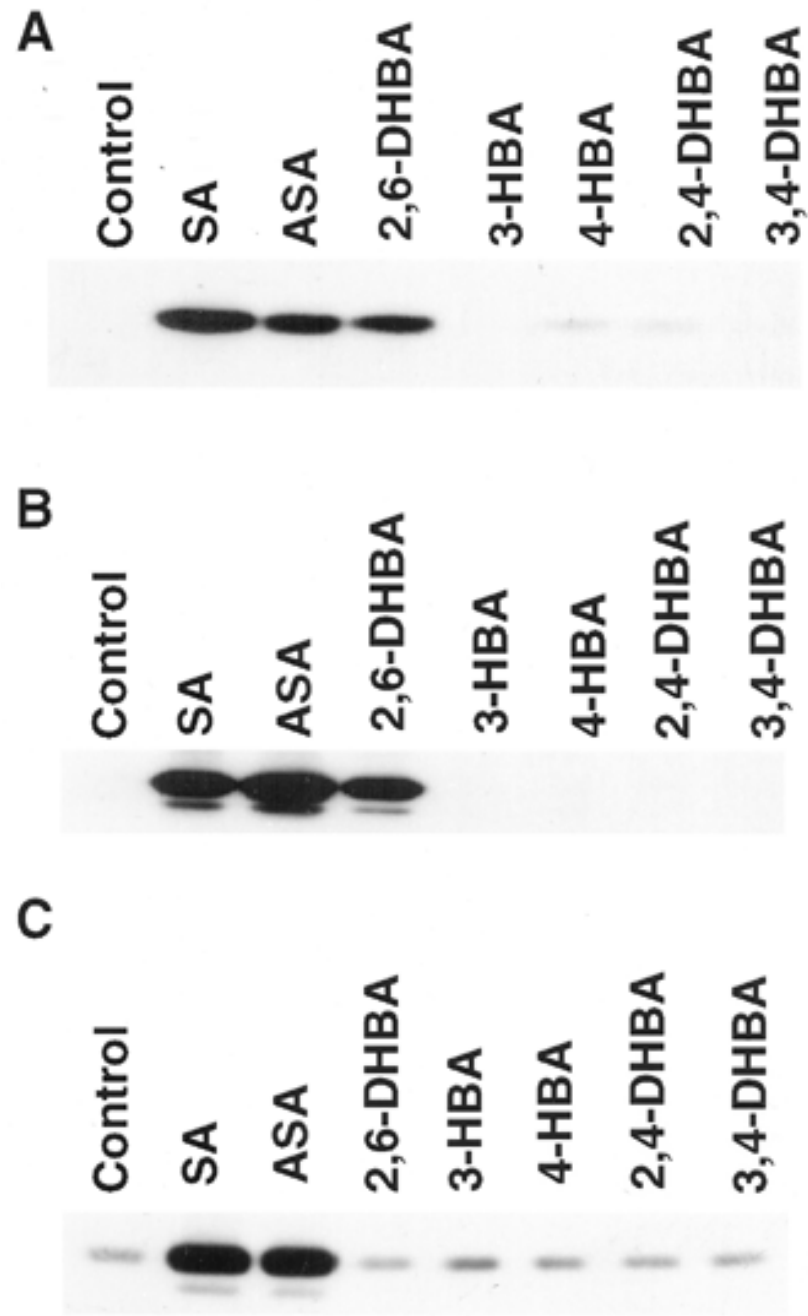

Fig. 2. Structural specificity for the induction of PR-1 protein accumulation by salicylic acid (SA) analogues in intact tobacco leaves and cell cultures. A, Tobacco leaves sprayed with $1 \mathrm{mM}$ of SA or its analogues. B, Tobacco leaf disks floated on $1 \mathrm{mM}$ of SA or its analogues. C, Tobacco cell cultures incubated with $50 \mu \mathrm{M}$ of SA or its analogues. SA analogues tested are acetylSA (ASA), 2,6-dihydroxybenzoic acid (2,6DHBA), 3-hydroxybenzoic acid (3-HBA), 4-hydroxybenzoic acid (4DHBA), 2,4-dihydroxybenzoic acid (2,4-DHBA) and 3,4-dihydroxybenzoic acid $(3,4-\mathrm{DHBA})$. Immunoblot analysis of PR-1 proteins was performed as described in Figure 1. phenolic compounds with high-performance liquid chromatography and quantitated photometrically (Hennig et al. 1993; Malamy et al. 1996). However, attempts to quantitate 2,6dihydroxybenzoic acid by a similar approach have failed because of undetectable fluorescence produced by the compound upon excitation with UV light of the same wavelength as used for SA detection. An indirect approach was, therefore, used to obtain information with regard to whether the failure of 2,6dichlorobenzoic acid to induce PR-1 protein accumulation in tobacco cell cultures was caused by its limited uptake and/or rapid metabolism.

We have previously demonstrated that SA and its biologically active analogues including 2,6-dihydroxybenzoic acid bind to and inhibit in vitro catalase activity of total soluble proteins isolated from tobacco plants (Chen et al. 1993). SA also inhibits in vivo catalase activity when incubated with tobacco cell cultures (Conrath et al. 1995). If the failure of 2,6dihydroxybenzoic acid to induce PR-1 protein accumulation in tobacco cell cultures was due to very limited uptake and/or rapid metabolism by tobacco cell cultures, it should be expected that incubation of tobacco cell cultures with the compound will not lead to effective inhibition of the in vivo activity of catalases even though the compound is capable of inhibiting the in vitro activity of isolated catalases. As shown in Table 1, both SA and acetylSA inhibited both the in vivo catalase activity of tobacco cell cultures and the in vitro catalase activity isolated from the same cultures as previously reported (Chen et al. 1993; Conrath et al. 1995). By contrast, 2,6-dihydroxybenzoic acid, while effective in inhibiting the in vitro catalase activity isolated from tobacco cell cultures, failed to inhibit the in vivo catalase activity of tobacco cell cultures (Table 1). Four biologically inactive analogues failed to inhibit both the in vivo and in vitro catalases activity of tobacco cell cultures (Table 1). These results suggest that the inability of 2,6-dihydroxybenzoic acid to induce PR-1 protein accumulation is probably due to its limited uptake and/or rapid metabolism by cell cultures.

In summary, the induction of PR-1 protein by SA and its analogues exhibited marked difference in both the dose re-

Table 1. Inhibition of catalase activity from tobacco cell cultures by salicylic acid (SA) analogues ${ }^{\mathrm{a}}$

\begin{tabular}{lcc}
\hline & \multicolumn{2}{c}{ Inhibition (\%) } \\
\cline { 2 - 3 } Compound & $\begin{array}{c}\text { Catalase activity } \\
\text { in vitro }\end{array}$ & $\begin{array}{c}\text { Catalase activity } \\
\text { in vivo }\end{array}$ \\
\hline SA & $75 \pm 8$ & $85 \pm 6$ \\
Acetyl SA & $68 \pm 8$ & $79 \pm 8$ \\
2,6-Dihydroxybenzoic acid & $73 \pm 6$ & $3 \pm 3$ \\
3-Hydroxybenzoic acid & $3 \pm 2$ & $2 \pm 2$ \\
4- Hydroxybenzoic acid & $2 \pm 1$ & $1 \pm 2$ \\
2,4-Dihydroxybenzoic acid & $4 \pm 3$ & $2 \pm 1$ \\
3,4-Dihydroxybenzoic acid & $2 \pm 3$ & $4 \pm 3$ \\
\hline
\end{tabular}

${ }^{a}$ For measuring in vitro catalase activity, soluble proteins from tobacco cell cultures were prepared by sonicating the cells in a homogenization buffer containing $20 \mathrm{mM}$ citrate, $\mathrm{pH} 6.5,5 \mathrm{mM} \mathrm{MgSO}_{4}, 1 \mathrm{mM}$ EDTA, $10 \%$ glycerol and $10 \mu \mathrm{g}$ of phenylmethylsulfonyl fluoride per $\mathrm{ml}$. The homogenate was clarified by centifugation and incubated with $1 \mathrm{mM}$ $\mathrm{SA}$ or its analogues. After $1 \mathrm{~h}$, catalase activity was assayed by measuring the $\mathrm{H}_{2} \mathrm{O}_{2}$-dependent $\mathrm{O}_{2}$ evolution with a commercial $\mathrm{O}_{2}$ electrode as previously described (Chen et al. 1997). For measuring in vivo catalase activity, tobacco cell cultures were incubated with $1 \mathrm{mM} \mathrm{SA}$ or its analogues. After $1 \mathrm{~h}$, the in vivo catalase activity of cell cultures was determined as previously described (Conrath et al. 1995). 
sponse and structural specificity between intact tobacco leaves and cell suspension cultures, which appeared to be caused largely by the difference in their uptake and/or metabolism. When assayed in tobacco cell cultures, the induction of PR-1 protein accumulation exhibited the same dose response and structural specificity with SA analogues as the potentiation of pathogen-induced hypersensitive disease resistance responses observed in soybean cell cultures. These results do not support the proposal that the potentiation of pathogen-induced hypersensitive disease resistance responses by SA involves a different signaling pathway that is more potent than the one(s) responsible for the induction of acquired resistance and PR gene expression. It should be noted that since assays for induction of PR-1 proteins and potentiation of pathogen signals by SA used different plant species as sources for the suspension culture cells, which could differ in both sensitivity and specificity to SA and its analogues, further experiments may be necessary to confirm the same dose response and structural specificity in the induction of these two important defense responses by SA in the same plant species.

\section{ACKNOWLEDGMENTS}

We would like to thank Allan Caplan for critically reading the manuscript. This work was supported by Idaho Agricultural Experimental Station, National Science Foundation EPSCoR grant EPS9350539 and US Department of Agriculture grant 96-36301-3316 (to Z. C.). Z. X. was supported by a plant biotechnology graduate assistantship from the University of Idaho Institute for Molecular and Agricultural Genetic Engineering.

\section{LITERATURE CITED}

Antoniw, J. F., and White, R. F. 1980. The effects of aspirin and polyacrylic acid on soluble leaf proteins and resistance to virus infection in five cultivars of tobacco. Phytopathol. Z. 98:331-341.

Baker, B., Zambryski, P., Staskawicz, B., and Dinesh-Kumar, S. P. 1997. Signaling in plant-microbe interactions. Science 276:726-733.

Baur, P., Hend, M., Schonhnerr, J., and Terence, G. B. 1997. Partition coefficients of active ingredients between plant cuticle and adjuvants as related to rates of foliar uptake. J. Agric. Food Chem. 45:36593665.

Cao, H., Glazebrook, J., Clarke, J. D., Volko, S., and Dong, X. 1997. The Arabidopsis NPR1 gene that controls systemic acquired resistance encodes a novel protein containing ankyrin repeats. Cell 88:57-63.

Chen, Z., Iyer, S., Caplan, A., Klessig, D. F., and Fan, B. 1997. Differential accumulation of salicylic acid and salicylic acid-sensitive catalase in different rice tissues. Plant Physiol. 114:193-201.

Chen, Z., Silva, H., and Klessig, D. F. 1993. Active oxygen species in the induction of plant systemic acquired resistance by salicylic acid. Science 262:1883-1886.

Conrath, U., Chen, Z., Ricigliano, J. W., and Klessig, D. F, 1995. Two inducers of plant defense responses, 2,6-dichloroisonicotinic acid and salicylic acid, inhibit catalase activity in tobacco. Proc. Natl. Acad. Sci. USA 92:7143-7147.

Hennig, J., Malamy, J., Grynkiewicz, G., Indulski, J., and Klessig, D. F. 1993. Interconversion of salicylic acid signal and its glucoside in tobacco. Plant J. 4:593-600.

Levine, A., Tenhaken, R., Dixon, R., and Lamb, C. 1994. $\mathrm{H}_{2} \mathrm{O}_{2}$ from the oxidative burst orchestrates the plant hypersensitive disease resistance response. Cell 79:583-595.

Malamy, J., Sánchez-Casas, P., Hennig, J., Guo, A., and Klessig, D. F. 1996. Dissection of the salicylic acid signaling pathway in tobacco. Mol. Plant-Microbe Interact. 9:474-482.

Ryals, J., Weymann, K., Lawton, K., Friedrich, L., Ellis, D., Steiner, H. Y., Johnson, J., Delaney, T. P., Jesse, T., Vos, P., and Uknes, S. 1997. The Arabidopsis thaliana NIM1 protein shows homology to the mammalian transcription factor inhibitor IкB. Plant Cell 9: 425-439.

Ryals, J. A., Neuenschwander, U. H., Willits, M. G., Molina, A., Steiner, H.-Y., and Hunt, M. D. 1996. Systemic acquired resistance. Plant Cell 8:1809-1819.

Shirasu, K., Nakajima, H., Krishnamachari, V., Dixon, R. A., and Lamb, C. 1997. Salicylic acid potentiates an agonist-dependent gain control that amplifies pathogen signals in the activation of defense mechanisms. Plant Cell 9:261-270.

Ward, E. R., Uknes, S. J., Williams, S. C., Dincher, S. S., Wiederhold, D. L., Alexander, D. C., Ahl-Goy, P., Metraux, J.-P., and Ryals, J. A. 1991. Coordinate gene activity in response to agents that induce systemic acquired resistance. Plant Cell 3:1085-1094.

Weymann, K., Hunt, M., Uknes, S., Neuenschwander, U., Lawton, K., Steiner, H., and Ryals J. 1995. Suppression and restoration of lesion formation in Arabidopsis Isd mutants. Plant Cell 7:2013-2022.

White, R. F. 1979. Acetylasalicylic acid (Aspirin) induces resistance to tobacco mosaic virus in tobacco. Virology 99:410-412

Yang, Y., Shah, J., and Klessig, D. F. 1997. Signal perception and transduction in plant defense responses. Gene Dev. 11:1621-1639. 